\title{
Experimentelle Studie zum Verständnis des bestimmten Artikels
}

\author{
Dagmar Bittner \\ ZAS Berlin \\ dabitt@zas.gwz-berlin.de
}

\begin{abstract}
Der Beitrag referiert Ergebnisse eines mit Erwachsenen durchgeführten Experiments zum Verständnis des bestimmten Artikels. Das Testmaterial entstammt einem für Kinder konzipierten Blickpräferenzexperiment. Die Durchführung des Tests mit Erwachsenen diente als Kontrolle der Verwendbarkeit der Materialien und der Überprüfung folgender Hypothese: Die referentielle Grundfunktion des Artikels besteht im Verweis auf begrenzte Ganze bzw. einen bestimmten (=begrenzten) Umfang einer Entität. Der interessante Aspekt des Experiments war, dass die Entscheidung zwischen [+begrenzt] vs. [-begrenzt] innerhalb einer pluralischen Kondition fallen musste, die Begrenztheitslesart wurde also nicht durch einzahlig auftretende zählbare Objekte erzeugt. Die Ergebnisse zeigen, dass die pluralische Kondition sich auf das Antwortverhalten der Probanden auswirkte. Probanden mit durchschnittlich längerer Reaktionszeit entscheiden sich anders als Probanden mit vergleichsweise kurzer Reaktionszeit. Während von der Gruppe mit spontanerem Entscheidungsverhalten die Hypothese im Hinblick auf den Artikel bestätigt wurde, scheint sich die Gruppe mit höheren Reaktionszeiten für das prototypischere Bild innerhalb der Pluralkondition zu entscheiden. ${ }^{1}$
\end{abstract}

\section{Ausgangspunkte und Hypothesen}

Kinder, die Deutsch als Muttersprache erwerben, beginnen im Alter von ca. 24 Monaten mit der Produktion des bestimmten Artikels. Fehler in der Setzung des Artikels sind von Beginn an ausgesprochen selten, so selten, dass sie als Ausnahmen eingeordnet werden können. Der Artikel wird zwar nicht sofort in allen erforderlichen Kontexten verwendet, doch wenn er erscheint, dann in den erwarteten zielsprachlichen Kontexten. Abweichungen von der Zielsprache treten lediglich im Hinblick auf die Wahl der richtigen Kasus-Genus-Form des Artikels auf. Die relativ hohe Sicherheit in der Setzung des Artikels (dies gilt auch für den unbestimmten Artikel, der hier nicht näher betrachtet wird) lässt vermuten, dass die Kinder ein bestimmtes Wissen über die referentiellen Kapazitäten des Artikels erworben haben, bevor sie mit seiner Produktion beginnen. Bei diesem Wissen muss es sich um die lexikalisch-funktionalen Informationsmerkmale handeln, die den bestimmten Artikel von allen anderen Modifizierern des Nomens unterscheiden.

Im Spracherwerb gehört der bestimmte Artikel zu den ersten grammatischen Einheiten, die im nominalen Bereich erworben werden. In seiner pronominalen Verwendung kann er sogar vor allen anderen grammatischen Einheiten (u.a. unbestimmter Artikel, Possessiv/Negationsartikel, Pluralflexion) erscheinen. Als Pronomen hat er ganz eindeutig ersetzende Funktion und wird auch vom Kind nur dann verwendet, wenn ein situativer oder anaphorischer Verweis zugrunde liegt. Dies gilt auch für die späteren Verwendungen innerhalb der Nominalphrase. Die primäre referentielle Funktion des bestimmten Artikels ist

Das Zustandekommen des Experiments ist der Mitarbeit von Franziska Bewer, Lydia Blenn, Milena Kühnast, Uwe Tautenhahn und Antje Schulz zu verdanken, die jeweils einen spezifischen Anteil an der Vorbereitung, der Durchführung und der statistischen Auswertung des Experiments haben. 
es, den Referenten als eine dem Hörer bekannte bzw. aus Ko- und/oder Kontext erschließbare Entität zu charakterisieren. Der Referent wird als (zuvor oder gerade) individualisiert dargestellt. Die Merkmale [+bekannt] und [+individualisiert] implizieren das Merkmal [+begrenzt]. Eine bekannte, individualisierte Entität ist ein in seinen Grenzen definiertes Ganzes. M.a.W., der bestimmte Artikel signalisiert die Referenz auf ein durch Ko- und/oder Kontext definiertes begrenztes Ganzes. (Sind keine individualisierenden Merkmale zugänglich, die den Referenten von allen anderen Elementen derselben lexikalischen Klasse unterscheiden, kann der bestimmte Artikel nur verwendet werden, wenn er generisch auf die gesamte Klasse, also das totale/maximale Ganze verweist, andernfalls ist er ungrammatisch.)

Die dem Experiment zugrundeliegende Hypothese kann somit folgendermaßen formuliert werden.

(1) Die grammatische Basisfunktion des bestimmten Artikels besteht in der Symbolisierung des perzeptuellen (=semantischen) Merkmals [+begrenzt]. der Referent des Nomens wird als begrenzte Entität charakterisiert.

Das Experiment ist Teil einer Untersuchung zur Bedeutung des perzeptuellen Merkmals [+begrenzt] in der grammatischen Strukturbildung und im Spracherwerb. Es wird angenommen, dass dieses Merkmal einen Startpunkt in einer hierarchisch organisierten Grammatik darstellt und dass die zuerst im Spracherwerb erscheinenden grammatischen Strukturen auf mit diesem Merkmal verbundene Oppositionen bezogen werden.

\section{Das Experiment}

\subsection{Aufbau}

Ablauf: Das Experiment beinhaltete die Zuordnung eines sprachlichen Stimulus zu einem von zwei simultan präsentierten Bildern. Die Probanden wurden am Laptop und unter Kopfhörern getestet. Dem Beginn des Experiments war eine Probe mit vier Bildpaaren vorgeschaltet, um Aufbau und manuelle Anforderung des Experiments kennen zu lernen. Die Aufgabe der Probanden bestand darin, durch Tastendruck anzuzeigen, welchem der beiden Bilder sie den gehörten sprachlichen Stimulus zuordnen würden. Im Falle der Zuordnung zum linken Bild war eine Taste auf der äußersten linken Seite der Tastatur zu drücken, im Falle der Zuordnung zur rechten Seite eine parallel liegende Taste auf der äußersten rechten Seite. Die einzelnen Bildpaare waren 2 Sekunden vor dem Ertönen des sprachlichen Stimulus und 5 Sekunden ab Ertönen des Stimulus zu sehen. Das Bildpaar verschwand, sobald der Proband durch Tastendruck eine Entscheidung eingegeben hatte. Hatte der Proband bis 4 Sekunden nach dem Einspiel des sprachlichen Stimulus keine Entscheidung abgegeben, ertönte ein dezenter Gong. Die Probanden wurden vor dem Experiment aufgefordert sich schnell und spontan zu entscheiden, der Gong sollte zusätzlich explizite Überlegensprozesse unterdrücken und durch den entstehenden Zeitdruck die bisher ausgebliebene Reaktion provozieren. (Im Experiment hat sich gezeigt, dass die Entscheidungen weitaus überwiegend vor dem Ertönen des Gongs getroffen wurden). Um eine Beeinflussung der Ergebnisse durch die Abfolge der Bildpaare ausschließen $\mathrm{zu}$ können, wurden diese in vier unterschiedlichen Abfolgen (Randomisierungen) dargeboten.

Testmaterial: Es wurden 16 Bildpaare präsentiert, die jeweils genau einen Gegenstand zeigten, der im Deutschen durch ein Konkretum bezeichnet wird. Der Gegenstand ist auf dem 
einen Bild zweimal abgebildet und auf dem anderen vielfach. Alle Bilder zeigen die Gegenstände vor unifarbenem Hintergrund und ohne Umgebung oder Zubehör, ${ }^{2}$ vgl. (2)

(2) Beispiel für Bildpaar
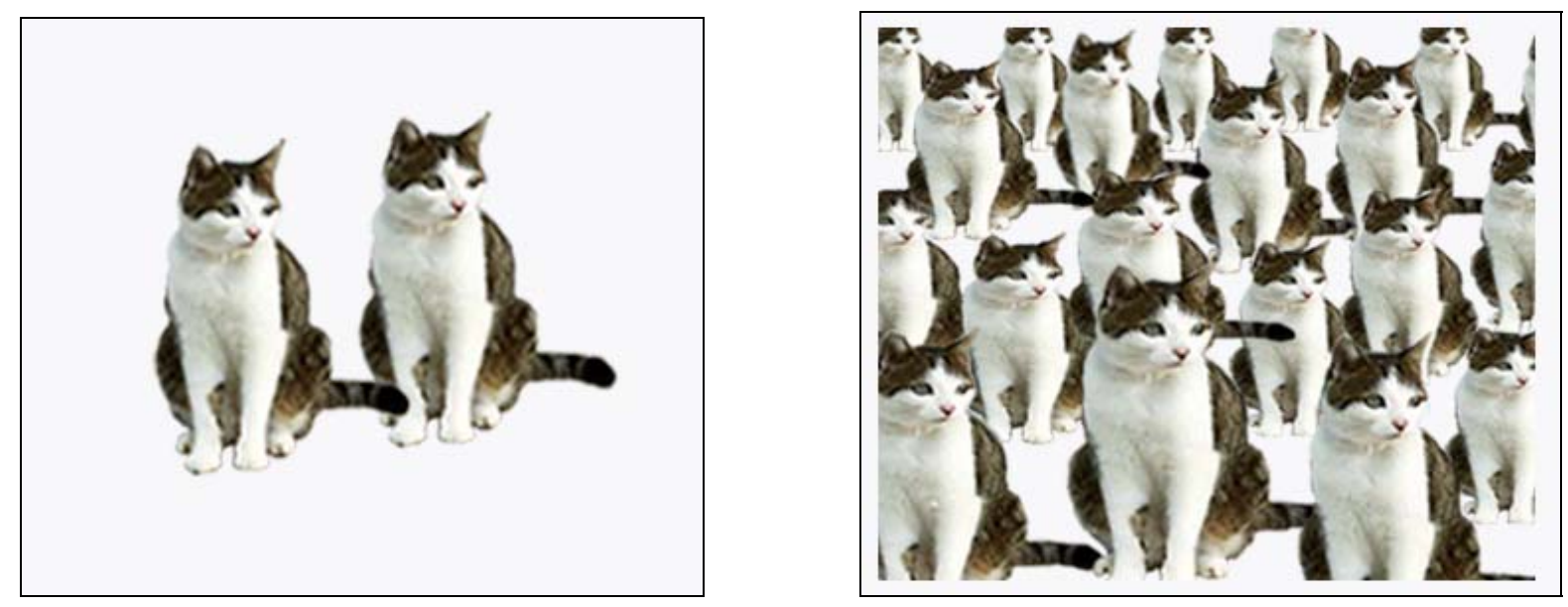

Die 16 im Test verwendeten Items sind:

(3) Affen, Bären, Bienen, Birnen, Blumen, Enten, Frauen, Hasen,

Jacken, Möhren, Katzen, Kerzen, Puppen, Scheren, Tassen, Uhren.

$\mathrm{Zu}$ jedem Bildpaar wurden zwei Sätze eingesprochen, die sich lediglich durch die An- oder Abwesenheit des Artikels die unterscheiden, also
(4) Schau mal, Affen
vs. Schau mal, die Affen
Schau mal, Kerzen
vs. Schau mal, die Kerzen ${ }^{3}$ usw.

Des weiteren wurden 8 Bildpaare mit Gegenständen, die durch Massennomen bezeichnet werden, präsentiert (Holz, Wasser, Honig, Milch, Gras, Marmelade, Saft, Sand). Diese Bildpaare wurden in analoger Weise gestaltet wie die der Konkreta, z.B. ein über den Bildrand hinausgehender Holzstapel vs. drei Scheite Holz oder eine über den Bildrand hinausgehende unruhige Wasseroberfläche vs. ein Glas Wasser. Auch die sprachlichen Stimuli waren analog zu denen der Konkreta gestaltet, also mit oder ohne Artikel, jedoch enthielten sie keine zweisilbigen auf -en gebildeten Pluralformen, weil Massennomen nicht pluralfähig sind, und neben dem Artikel die kamen auch der (Honig) und das (Wasser) vor. Ziel der Hinzufügung dieser Bilder war einerseits, das Durchschauen der Fragestellung des

2 Die Bilder, es handelt sich teilweise um Fotografien und teilweise um kolorierte Zeichnungen, wurden so ausgewählt, dass sie möglichst prototypische Exemplare darstellen und somit gut erkennbar sind. Sie wurden aus dem Internet heruntergeladen oder eingescannt, mit dem Programm Photoshop bearbeitet und als .jpg-Dateien abgespeichert. Aus technischen Gründen, die die Präsentation per Beamer betreffen, wurden alle Bilder im Format 300x300 Pixel angefertigt. Zur Herstellung der Bildpaare wurde die jeweilige Abbildung entweder zweifach oder vielfach auf dem Bild angeordnet. Die vielfach-Bilder sind grundsätzlich so gestaltet, dass die mittigen Objekte vollständig zu sehen sind, während diejenigen an den Bildrändern teilweise abgeschnitten sind.

3 Die Sätze wurden in einer schalldichten Tonkabine mehrfach eingesprochen und mit einem Sony-DATrecorder aufgenommen. Die Sprecherin äußerte die Sätze in einer hellen und freundlichen, kindgerichteten Art. Das so gewonnene Material wurde im Computer digitalisiert. Mit dem Programm Cool Edit wurden die Sätze einzeln ausgeschnitten und als .wav-Dateien gespeichert. Aus den mehrfach vorhandenen Sätzen wurde das jeweils beste Exemplar ausgewählt. 
Experiments und damit den Aufbau einer bewussten Antwortstrategie zu erschweren, und andererseits, zu überprüfen, ob sich ein analoger Test mit Massennomen anbietet.

Präsentation: $\mathrm{Zu}$ jedem Bildpaar wurde genau einer der beiden Sätze dargeboten. Es wurde darauf geachtet, dass jedem Bild über alle Randomisierungen 2x der Stimulus mit und 2x der Stimulus ohne Artikel zugeordnet wurde, dass zwei Randomisierungen beim ersten Bildpaar einen Stimulus ohne und zwei einen Stimulus mit Artikel aufwiesen und insgesamt ein ausgewogenes Verhältnis in der Verteilung der beiden Stimulustypen vorliegt. Auch die Platzierung der Bilder wurde ausbalanciert. Jeder Bildtyp (zwei vs. viele Gegenstände) wurde $12 \mathrm{x}$ rechts und $12 \mathrm{x}$ links präsentiert.

\subsection{Probanden}

Das Experiment wurde in Berlin am Zentrum für Allgemeine Sprachwissenschaft, Universalienforschung und Typologie durchgeführt. Es nahmen 38 Probanden im Alter von 18 bis 65 Jahren teil, die mit Deutsch als Muttersprache aufgewachsen waren und keine gesundheitlichen, insbesondere keine Hörprobleme hatten. Von der Endauswertung wurden die Probanden ausgeschlossen, die in den 16 Bildpaaren mit Konkreta zu über 75\% ein und denselben Bildtyp gewählt haben. Dies waren 5 Probanden mit einer Präferenz für den zweiund 9 Probanden mit einer Präferenz für den viele-Bildtyp. Eine Präferenz die rechte oder linke Taste zu drücken konnte nicht festgestellt werden. Die Auswertung umfasst somit 24 Probanden.

\section{Erwartungen}

Wie deutlich wurde, ist das Experiment so angelegt, dass sich die Bilder lediglich durch die Präsentation von zwei vs. vielen Objekten bzw. einer begrenzten und einer unbegrenzten Menge unterscheiden und die sprachlichen Stimuli lediglich durch die An- oder Abwesenheit des bestimmten Artikels. Die zwei-Bilder präsentieren eine klar als begrenzt erfassbare Menge, insbesondere wenn sie einer Menge mit unbestimmt vielen Objekten gegenübergestellt ist. Der Eindruck der Unbegrenztheit der viele-Bilder wird nicht nur durch die größere Anzahl der Objekte erzeugt, sondern vor allem durch die über den Bildrand hinausweisenden abgeschnittenen Objekte.

Entsprechend der Hypothese in (1) wurde erwartet, dass die Verbindung des bestimmten Artikels mit dem Merkmal [+begrenzt] durch folgendes Verhalten offensichtlich wird: Nach dem Ertönen des Satzes mit Artikel (Schau mal, die Enten) drückt der Proband die Taste für das Bild mit den zwei Objekten, denn nur dieses Bild präsentiert eine begrenzte Entität. Bei den Sätzen ohne Artikel (Schau mal, Enten) wird keine Präferenz für eines der beiden Bilder erwartet, da auf beiden Bildern mehrere Objekte zu sehen sind, der unspezifische pluralische Stimulus also auf beide Bilder zutrifft. Für die Bilder mit den Massennomen-Gegenständen bestand im Grunde eine analoge Erwartung, jedoch war das Material hier weitaus heterogener, so dass eher auf den Ablenkeffekt dieser Bildpaare gesetzt wurde

\section{$4 \quad$ Ergebnisse}

Angeboten wurden zwei Bildtypen und zwei Stimulustypen. Dies ergibt vier mögliche Kategorien für die Entscheidungen der Probanden: ,zwei-Bild bei mit-Artikel-Satz', zweiBild bei ohne-Artikel-Satz', viele-Bild bei mit-Artikel-Satz' und ,viele-Bild bei ohne-ArtikelSatz'. Ausgewertet wurde zum einen die Häufigkeit der Entscheidungen für eine der 4 
Kategorien. Zum anderen wurden die Reaktionszeiten innerhalb dieser Kategorien betrachtet und ein Vergleich der Gruppe mit den niedrigeren Reaktionszeiten und der Gruppe mit den höheren Reaktionszeiten vorgenommen. Als erstes wurden aber die Ergebnisse für die Massennomen gesondert von den Ergebnissen für die Konkreta betrachtet.

\subsection{Massennomen}

Wie erwartet zeigte sich kein spezifisches Antwortmuster in diesem Teil des Experiments. Innerhalb der Kondition ,mit-Artikel-Satz' (Schau mal, das Holz) wurde 49 mal das Bild mit der begrenzten Menge gewählt und 43 mal das Bild mit der unbegrenzten Menge. In der Kondition ,ohne-Artikel-Satz' (Schau mal, Holz) wurde 50 mal das Bild mit der begrenzten und 45 mal das Bild mit der unbegrenzten Menge gewählt. ${ }^{4}$ Die Art des Stimulus erzeugt also keinerlei Präferenzen für die Wahl eines der beiden Bilder. Es kann davon ausgegangen werden, dass diese Bilder als Ablenker wirkten und den Aufbau einer Antwortstrategie für die Konkreta-Bilder erschwert haben.

\subsection{Konkreta}

Aus der Multiplikation von 24 Probanden und 16 Konkreta-Bildpaaren ergibt sich, dass der Auswertung 192 Sätze mit und 192 Sätze ohne Artikel zu Grunde liegen. Ausgewertet wurden das Entscheidungsverhalten und die Reaktionszeiten der Probanden. Die Ergebnisse wurden durch Anova- und T-Test-Berechnung auf ihre statistische Signifikanz überprüft.

\subsubsection{Entscheidungsverhalten über alle 16 Bildpaare}

Der Satz mit Artikel wurde in 58,2\% aller Fälle dem zwei-Bild zugeordnet, der Satz ohne Artikel in 62,8\% aller Fälle dem viele-Bild (vgl. Tabelle 1, letzte Zeile). Der T-Test ergibt für die mit-Artikel-Sätze nur eine schwache Tendenz, bevorzugt den zwei-Bildern zugeordnet zu werden $(p<0,194)$. Dagegen ist die Zuordnung der ohne-Artikel-Sätze zum viele-Bild eine signifikante Korrelation $(\mathrm{p}<0,023)$.

\subsubsection{Entscheidungsverhalten nach Phasen}

Um das Verhalten der Probanden im Verlauf des Experiments zu verfolgen, wurde eine Phaseneinteilung in Anfangs-, Mittel- und Endphase des Experiments, vorgenommen. Jede der 3 Phasen umfasst 8 Bildpaare, die sowohl Konkreta- als auch Massennomen-Bildpaare enthalten. Ausgewertet wurden nur die Konkreta-Bildpaare. Tabelle 1 präsentiert die Ergebnisse für jede der 3 Phasen.

Tabelle 1: Entscheidungsverhalten in Anfangs-, Mittel- und Endphase (+ Gesamtexperiment)

\begin{tabular}{|l|c|c|c|c|}
\hline \multirow{2}{*}{} & \multicolumn{2}{|c|}{ mit Artikel-Kondition } & \multicolumn{2}{c|}{ ohne Artikel-Kondition } \\
\cline { 2 - 5 } & zwei & viele & zwei & viele \\
\hline Phase I & $48 \%$ & $52 \%$ & $41 \%$ & $59 \%$ \\
\hline Phase II & $\mathbf{6 6 \%}$ & $33 \%$ & $40 \%$ & $60 \%$ \\
\hline Phase III & $60 \%$ & $39 \%$ & $27 \%$ & $\mathbf{7 2 \%}$ \\
\hline Gesamtexp. & $58 \%$ & $41 \%$ & $37 \%$ & $63 \%$ \\
\hline
\end{tabular}

4 Die Differenzen in den Summen für beide Konditionen ergeben sich durch Zeitüberschreitungen im Experiment, d.h. in insgesamt 5 Fällen wurde bei einem Massennomen-Bildpaar keine Entscheidung abgeben. 
Die dreifache Anova über die Faktoren Artikel-Objektanzahl-Phase ergibt zwar keinen Haupteffekt, jedoch eine signifikante Interaktion zwischen den Faktoren Artikel und Objektanzahl $(\mathrm{F}=6,099, \mathrm{p}<0,021)$. Zugleich zeigt sich eine Tendenz, dass der Grad der Interaktion der beiden Faktoren durch den Faktor Phase beeinflusst wird $(p<0,088)$. Die in Tabelle 1 gegebenen Prozentzahlen zum Entscheidungsverhalten der Probanden zeigen, dass in der Anfangsphase eine leichte Tendenz besteht, den sprachlichen Stimulus mit dem vieleBild zu verbinden, egal ob dieser einen Artikel enthält oder nicht. Statistisch gesehen ist das Verhalten in dieser Phase jedoch zufällig. In der Mittelphase wird für den mit-Artikel-Satz eindeutig das zwei-Bild favorisiert. Die Korrelation ist hier statistisch hochsignifikant $(p<0,012)$. Für den ohne-Artikel-Satz zeigt sich zwar eine zunehmende Tendenz zur Zuordnung zum viele-Bild, sie bleibt jedoch statistisch noch zufällig $(p<0,146)$. In der Endphase wird die Zuordnung des mit-Artikel-Satzes zum zwei-Bild wieder schwächer und rutscht unter das Signifikanzlevel. Gleichzeitig steigt die Zuordnung des ohne-Artikel-Satzes zum viele-Bild weiter an, so dass diese Korrelation nun statistisch hochsignifikant wird $(p<0,002)$.

\subsubsection{Reaktionszeiten}

Die Messung der Reaktionszeiten startete bei der Präsentation eines mit-Artikel-Satzes, mit dem Beginn der Artikelartikulation (also mit beginn des /d/-Lautes) und bei den ohne-ArtikelSätzen mit der Artikulation des ersten Lautes des Substantivs.

Es wurden zum einen die Mittelwerte (MW) der Reaktionszeiten (RZ) für die gesamte Gruppe ermittelt. Zum anderen wurden die 24 Probanden in zwei Gruppen unterteilt: a) die Gruppe der 12 Probanden mit den über den Gesamtverlauf des Experiments (also einschließlich der Massennomen) niedrigeren RZ (NiRZ), und b) die alternative Gruppe der 12 Probanden mit den höheren RZ (HoRZ).

Tabelle 2: Reaktionszeiten (MW in ms)

\begin{tabular}{|l|l|l|l|l|}
\hline & \multicolumn{2}{|c|}{ mit Artikel-Kondition } & \multicolumn{2}{c|}{ ohne Artikel-Kondition } \\
\hline & zwei & viele & zwei & viele \\
\hline Gesamtgruppe & 2101 & 2162 & 1973 & 2089 \\
\hline NiRZ-Gruppe & 1940 & 1870 & 1822 & 1839 \\
\hline HoRZ-Gruppe & 2281 & 2433 & 2179 & 2301 \\
\hline
\end{tabular}

Die RZ ist in allen 3 Probandenkonstellationen kürzer, wenn ein Satz ohne Artikel gehört wurde, als wenn ein Satz mit Artikel gehört wurde. Berechnet wurde nur die statistische Signifikanz für die Gesamtgruppe $(\mathrm{F}=10,412, \mathrm{p}>0,004)$. Eine Berechnung der $\mathrm{RZ}$ in den einzelnen Phasen zeigt, dass dieser Unterschied in den beiden ersten Phasen des Experiments sehr ausgeprägt ist $(p<0,023 / 0,036)$, sich aber in der Endphase des Experiments verliert $(\mathrm{p}<0,839)$. Für das Gesamtexperiment ergibt sich aus Tabelle 2, dass dieser Unterschied in der HoRZ-Gruppe weit ausgeprägter ist als in der NiRZ-Gruppe.

Nur in der HoRZ-Gruppe ist ein Unterschied in der Entscheidungsdauer für das zwei- oder viele-Bild innerhalb der beiden Satztypen (mit/ohne-Artikel) festzustellen. In beiden Konditionen ist die RZ kürzer, wenn das zwei-Bild zugeordnet wird. Für die Gesamtgruppe liegt kein statistisch signifikanter Unterschied vor, was daraus resultiert, dass es in der NiRZGruppe kaum RZ-Differenzen zwischen den 4 Kategorien gibt. 


\subsubsection{Entscheidungsverhalten der beiden Reaktionszeit-Teilgruppen}

Der sich andeutende Unterschied im Verhalten der beiden nach RZ unterschiedenen Probandengruppen wurde zum Anlass genommen, auch das Entscheidungsverhalten dieser beiden Gruppen nochmals separat zu untersuchen. Eine statistische Auswertung wurde aufgrund der geringen Gruppenstärke nicht vorgenommen.

Tabelle 3: Entscheidungsverhalten der NiRZ-Gruppe

\begin{tabular}{|l|c|c|c|c|}
\hline \multirow{2}{*}{} & \multicolumn{2}{|c|}{ mit Artikel-Kondition } & \multicolumn{2}{c|}{ ohne Artikel-Kondition } \\
\cline { 2 - 5 } & zwei & viele & zwei & viele \\
\hline Gesamtexperiment & $\mathbf{6 0 \%}$ & $39 \%$ & $43 \%$ & $57 \%$ \\
\hline Phase I & $50 \%$ & $50 \%$ & $48 \%$ & $52 \%$ \\
\hline Phase II & $\mathbf{6 8 \%}$ & $32 \%$ & $44 \%$ & $56 \%$ \\
\hline Phase III & $\mathbf{6 5 \%}$ & $35 \%$ & $34 \%$ & $\mathbf{6 6 \%}$ \\
\hline
\end{tabular}

Tabelle 4: Entscheidungsverhalten der HoRZ-Gruppe

\begin{tabular}{|l|c|c|c|c|}
\hline \multirow{2}{*}{} & \multicolumn{2}{|c|}{ mit Artikel-Kondition } & \multicolumn{2}{c|}{ ohne Artikel-Kondition } \\
\cline { 2 - 5 } & zwei & viele & zwei & viele \\
\hline Gesamtexperiment & $56 \%$ & $44 \%$ & $32 \%$ & $\mathbf{6 8} \%$ \\
\hline Phase I & $48 \%$ & $52 \%$ & $37 \%$ & $\mathbf{6 3} \%$ \\
\hline Phase II & $\mathbf{6 5} \%$ & $35 \%$ & $35 \%$ & $\mathbf{6 5} \%$ \\
\hline Phase III & $55 \%$ & $45 \%$ & $24 \%$ & $\mathbf{7 6} \%$ \\
\hline
\end{tabular}

Generell gilt: Wenn ein Bildtyp bevorzugt zugeordnet wird, ist es im Fall des mit-ArtikelSatzes stets das zwei-Bild und im Fall des ohne-Artikel-Satzes stets das viele-Bild. Darüber hinaus verhalten sich beide Gruppen aber nahezu konträr. Die HoRZ-Gruppe zeigt von Beginn an eine deutliche Präferenz den ohne-Artikel-Satz mit dem viele-Bild zu verbinden. Für den mit-Artikel-Satz hat sie (ausgenommen Phase II) keine Vorlieben. Die NiRZ-Gruppe präferiert dagegen ab Phase II gerade für den mit-Artikel-Satz einen Bildtyp, und zwar das zwei-Bild. Diese Korrelation wird von Phase zu Phase stärker. Für den ohne-Artikel-Satz wird erst in der Endphase des Experiments eine Präferenz für das viele-Bild sichtbar.

\section{Diskussion der Ergebnisse}

Zunächst kann festgestellt werden, dass der entwickelte Experimentaufbau es ermöglicht, eine unterschiedliche Beurteilung von Nominalphrasen (NP) mit-/ohne-Artikel im Bereich der Konkreta sichtbar zu machen. In einer binären Entscheidungssituation wird eine Nominalphrase mit Artikel bevorzugt dem Bild zugeordnet, welches eine begrenzte Menge, d.h. das Merkmal [+begrenzt], präsentiert. Demgegenüber wird eine NP ohne Artikel bevorzugt dem Bild zugeordnet, welches eine unbegrenzte Menge präsentiert.

Die Reaktionszeitunterschiede zwischen den Konditionen mit-Artikel-Satz vs. ohne-ArtikelSatz zeigen, dass die mit dem Auftreten des Artikels verbundene Komplexitätssteigerung in der Struktur der Äußerung mit einer längeren Verarbeitungsphase korrespondiert. Wie Tabelle 2 zeigt, liegen die Mittelwerte der Reaktionszeiten in der ohne-Artikel-Kondition stets unter den Mittelwerten der mit-Artikel-Kondition (mit einer Ausnahme, der Zuordnung des ohneArtikel-Satzes zu den viele-Bildern in der HoRZ-Gruppe; dazu unten). Die Komplexitäts- und 
Anforderungserhöhung durch den Artikel spiegelt sich auch bei den Zeitüberschreitungen, also den Fällen, in denen keine Entscheidung für eines der beiden Bilder abgegeben wurde. Es traten 9 solche „Ausfälle“ auf, in 7 davon war der sprachliche Stimulus ein Satz mit Artikel. Da alle anderen Eigenschaften des Stimulus stets unverändert blieben, kann die längere RZ bei Artikelsätzen nur auf den höheren zu verarbeitenden Informationsanteil durch den Artikel zurückgeführt werden. Ein Einfluss der mit dem Artikel verbundenen längeren Dauer des sprachlichen Stimulus ist ebenfalls möglich, sollte jedoch, da die Probanden die Gegenstände sahen, die mit dem Nomen bezeichnet wurden, weniger stark ins Gewicht fallen und letztlich kein Grund für Zeitüberschreitungen sein. ${ }^{5}$

$\mathrm{Zu}$ beachten ist das unterschiedliche Verhalten der Probanden, die sich spontan (NiRZ) und der Probanden, die sich mit einer gewissen Verzögerung (HoRZ) entschieden haben. Die NiRZ-Gruppe zeigt nahezu vollständig das erwartete Ergebnis: die NP ohne Artikel provoziert keine eindeutige Entscheidung zwischen den beiden Bildern; die NP mit Artikel provoziert eine Entscheidung zugunsten der begrenzten Menge, also des Merkmals [+begrenzt]. Erst nachdem sich die Zuordnung Artikel-begrenzte Menge herausgebildet hat, wird in Phase III die Opposition artikellose NP-unbegrenzte Menge gebildet. Möglicherweise konnten die Probanden gegen Ende des Experiments eine Bewusstheit über die implementierte Opposition erlangen und eine Antwortstrategie entwickeln. Interessant ist, dass während nun die Leistung für die oppositionelle Korrelation ,ohne-Artikel-unbegrenzte Menge' deutlich besser wird, die Leistung für die zuvor etablierte Korrelation absinkt. Die Konzentration liegt in Phase III deutlich auf dem neu erfassten Zusammenhang. Ganz parallele Verläufe sind auch im natürlichen Spracherwerb beobachtet worden. Insgesamt stützt das Ergebnis das Vorhaben eines analogen Blickpräferenzexperiments mit Kindern, da auch dieses auf einer spontanen Zuordnung zwischen visuellem und auditivem Stimulus aufbaut. Vor allem aber bestätigt das Verhalten dieser Probandengruppe die unter (1) formulierte Hypothese zur referentiellen Funktion des bestimmten Artikels. Er informiert den Hörer darüber, dass der Referent des Nomens ein begrenztes Ganzes darstellt.

Die Probanden der HoRZ-Gruppe scheinen das Experiment insgesamt reflektierter und zugleich unsicherer absolviert zu haben. Wenngleich ihre Präferenz für die Zuordnung des ohne-Artikel-Satzes zum viele-Bild bereits in Phase I sichtbar wird und sich im Verlauf des Experiments noch verstärkt, zeigt ein Vergleich der RZen, dass diese gerade bei der Zuordnung der viele-Bilder besonders stark über denen der NiRZ-Gruppe liegen (vgl. Tabelle 2, >460ms im MW) - dies wiederum besonders massiv in der mit-Artikel-Kondition ( $>560 \mathrm{~ms})$. Die Wahl des viele-Bildes scheint eher nach einiger „Überlegung“ und unter dem Zwang eine Entscheidung fällen zu müssen, erfolgt zu sein. Sie kann als schließliche Entscheidung für die "sichere Seite“ interpretiert werden. Im Versuch eine bewusste Entscheidung zu treffen, geraten die Probanden in den Konflikt zwischen Plural-Information und Artikel-Information. Während die Mehrzahligkeit in den Stimuli objektiv gegeben ist, stellt die Begrenztheits-Information des Artikels eine zusätzliche, d.h. optionale Möglichkeit der Betrachtung der angebotenen Referenten dar und ist entsprechend schwieriger zu erfassen. Scheitert die Interpretation der Artikel-Information bleibt nur die pluralische Information und hier ist das viele-Bild der quasi prototypischere Kandidat.

Ein zusätzliches Indiz für die stärkere Unsicherheit im Entscheidungsverhalten der HoRZGruppe ergibt sich bei einem Blick auf die „Ausfälle“. In der NiRZ-Gruppe traten nur zwei Zeitüberschreitungen auf, beide bei einem Massennomen-Bildpaar und mit-Artikel-Satz. In

Man würde in diesem Fall vor allem „Ausfälle“ bei den mehrsilbigen Stimuli und vor allem bei dem dreisilbigen marmelade erwarten. Der Marmelade-Stimulus war jedoch nur 1x unter den „Ausfällen“, dagegen finden sich hier 4 Einsilber (3x saft, 1x holz). 
der HoRZ-Gruppe waren es 7 Zeitüberschreitungen davon 4 bei einem Kontinua-Bildpaar (3 mit-, 1 ohne-Artikel-Satz). Neben der erwartbar höheren Zahl an Zeitüberschreitungen in der HoRZ-Gruppe sind hier nun auch und in gleichem Maße wie Massennomen-Bildpaare Kontinua-Bildpaare betroffen und eben besonders in der mit-Artikel-Kondition.

Schließlich, indirekt spricht die klarere Zuordnung der ohne-Artikel-Sätze zum viele-Bild gegenüber der Unentschiedenheit in der Zuordnung der mit-Artikel-Sätze dafür, dass für letztere eine Zuordnung zum zwei-, also [+begrenzt]-Bild viel wahrscheinlicher ist als für erstere. Die stärkere Reflexion des Experiments hat die entsprechende Entscheidung offensichtlich aufgrund der im gegebenen Experimentaufbau „nur“ zusätzlichen Funktion des Artikels weitgehend unterdrückt.

Abschließend lässt sich sagen, dass das Experiment eine Bestätigung der Hypothese erbracht hat, dass die referentielle Basisfunktion des bestimmten Artikels in der Symbolisierung des Merkmals [+begrenzt] liegt. Das unterschiedliche Verhalten der Probandengruppe mit den niedrigeren vs. der mit den höheren Reaktionszeiten legt nahe zur Kontrolle dieses Ergebnisses ein analoges aber ausschließlich für Erwachsene konzipiertes Experiment mit höherem Reaktionszeitdruck durchzuführen. 